\title{
KNOWLEDGE, FALSE BELIEF, AND REDUCTIO
}

\author{
MATT LEONARD
}

Inquiry (Forthcoming)

\begin{abstract}
Recently, a number of cases have been proposed which seem to show that - contrary to widely held opinion - a subject can inferentially come to know some proposition $p$ from an inference which relies on a false belief $q$ which is essential. The standard response to these cases is to insist that there is really an additional true belief in the vicinity, making the false belief inessential. I present a new kind of case suggesting that a subject can inferentially come to know a proposition from an essential false belief where no truth in the vicinity seems to be present.
\end{abstract}

Until recently, it was widely held that knowledge cannot be generated from false belief,

One cannot inferentially come to know a proposition $p$ if one's inference relies on a false belief $q$ which is essential.

(No FAlse Belief)

where a false belief is essential just in case it figures in all the belief-forming processes that generate the relevant belief. ${ }^{1}$ However, there have been a handful of alleged counterexamples proposed in the literature. Here are two from Warfield (2005).

BAD Counting: Counting with some care the number of people present at my talk, I reason: 'There are 53 people at my talk; therefore, my 100 handout copies are sufficient'. My premise is false. There are 52 people in attendance. I double counted one person who changed seats during the count. And yet I know my conclusion.

DodGy WATCH: I have a 7PM meeting and extreme confidence in the accuracy of my fancy watch. Having lost track of the time and wanting to arrive on time for the meeting, I look carefully at my watch. I reason:

\footnotetext{
${ }^{1}$ For instance, Russell (1912, p. 76), Clarke (1963), Armstrong (1973: p. 152), Williamson (2007, pgs. 145-7), and Kripke (2011). There are a number of different conceptions of what it is to rely on a belief that is essential. Here I follow Hawthorne and Rabinowitz (2018). However, later in the paper, I will consider another plausible conception from Klein (2008).
} 
'It is exactly 2:58PM; therefore, I am not late for my 7PM meeting'. I know my conclusion, but as it happens it's exactly 2:57PM, not 2:58PM.

At first glance, it looks like these cases - which Warfield calls cases of knowledge from falsehood, or KFF cases - are straightforward counterexamples to (No FAlse Belief). In each case, it looks like I come to know a proposition from an inference which relies on a false belief that is essential. ${ }^{2}$

There have been a handful of responses to these sorts of cases by defenders of (No False Belief): Ball and Blome-Tillmann (2014), Montminy (2014), Schnee (2015), for instance. Though there are some subtle differences in their accounts, the underlying idea is basically the same and is what Fitelson (2018) calls the standard response. The standard response exploits an incredibly natural observation about KFF cases: in each of the cases, there is actually a truth in the vicinity the subject believes (or dispositionally believes) from which the subject infers the relevant conclusion. If this is the case, then the false belief made explicit in each of the cases (viz., 'there are 53 people present' and 'it is exactly $2: 58 \mathrm{PM}$ ') is not really essential to the inference. And so the cases are not counterexamples to (No FALSE BELIEF), after all. ${ }^{3}$

I am sympathetic to the standard response. ${ }^{4}$ However, in this paper, I present a new kind of counterexample to (No FALSE BELIEF). Unlike the cases above, this case involves a subject inferentially coming to know a proposition from a false belief where there genuinely seems to be no relevant truth in the vicinity. This suggests that even if the standard response has a diagnosis for the more familiar KFF cases in the literature, there are additional counterexamples to which it seems to offer no help. In the first part of the paper, I briefly sketch Ball and Blome-Tillmann's account, since it is one of the more developed versions of the standard response. In the second part of the paper, I present the new case and then suggest why the standard response doesn't help.

\section{KFF CASES And Truths in the Vicinity}

Ball and Blome-Tillmann (2014) claim that KFF cases are more accurately described as cases of knowledge despite falsehood (or KDF cases). ${ }^{5}$ They offer

\footnotetext{
${ }^{2}$ And thus, the cases also seem to be counterexamples to the related principle of counterclosure, which says that one cannot inferentially come to know a proposition from an inference relying on unknown premises. See Luzzi (2010).

${ }^{3}$ In fact, one of the leading voices on the other side of the debate also relies on such an observation. Klein (2008), who takes KFF cases to be counterexamples to something like (No FALSE BeliEf), attempts to give a definition of such cases, which he calls useful falsehoods. For Klein, one necessary condition of a KFF case is that there is some truth in the vicinity that entails the conclusion.

${ }^{4}$ Though see Buford and Cloos (2017) and Fitelson (2018) for some objections.

${ }^{5}$ As does Montminy (2014).
} 
the following diagnosis of what is actually going on in the cases. In each of the cases, where $S$ is the subject, $p$ is the false belief, and $q$ is the known conclusion, there are two propositions $t_{1}$ and $t_{2}$ (where $t_{2}$ is the so-called 'truth in the vicinity') such that:

(1) $t_{1}$ evidentially supports both $p$ and $t_{2}$ for $S$.

(2) $t_{2}$ is entailed by $p$.

(3) $S$ knows both $t_{1}$ and $t_{2}$.

(4) $S$ 's belief that $q$ is properly based on her knowledge that $t_{2}$.

Ball and Blome-Tillmann then propose the values of these variables for our two cases:

\section{Bad Counting}

$t_{1}$ : The result of my count was ' 53 '.

$t_{2}$ : There are 53 people at my talk give or take a few.

$p$ : There are 53 people in the room.

$q$ : I have enough handouts.

\section{DODGY WATCH}

$t_{1}$ : My watch reads ' $2: 58$ '.

$t_{2}$ : It's approximately 3PM.

$p$ : It's $2: 58$.

$q$ : I'm not late for my meeting.

In each of the cases, there is a truth in the vicinity (viz., 'There are 53 people at my talk give or take a few' and 'It's approximately 2:58PM') that is both believed and known, which renders the false belief inessential. Fitelson (2018) calls this additional belief the proxy.

Advocates of the standard response go a step further and ask us to really consider versions of BAD COUNTING and DoDGY WATCH where the proxies are explicitly lacking. In these cases, it seems less obvious that the subject comes to know the relevant proposition. As Schnee (2015) argues, perhaps these stripped down versions of the cases are really just Gettier cases. I am sympathetic to this diagnosis of BAD Counting and Dodgy Watch.

\section{An Unintended Reductio}

However, consider the following semi-fictional case.

EuCliD's EPIPHANY: In his teenage years, Euclid loved and knew a great deal about logic, but not mathematics. He loved proving things 
by reductio, but could care less about the natural numbers. Though he knew what a prime number was, he believed that there were only finitely many of them. One day, Euclid woke up with an interest in math and reasoned as follows: 'There are only finitely many primes. So let $N$ be the product of all primes $\left(p_{1} \times p_{2} \times \ldots \times p_{n}\right) . \quad N+1$ is its successor $\left(p_{1} \times p_{2} \times \ldots \times p_{n}+1\right)$. Now let $p$ be some prime among $p_{1}, \ldots, p_{n}$ which divides $N+1$. But since $p$ is a prime, it also divides $N$, and thus it divides the difference between $N$ and $N+1$. But the difference is 1 : that's impossible! So $p$ is yet another prime, not one of $p_{1}, \ldots, p_{n}$. But that's impossible! There must be an infinite number of primes.'

A few initial remarks about the case. First, not all arguments from reductio are counterexamples to (No FALSE BELIEF). Typically, when one attempts to prove some proposition by reductio, one does not falsely believe the assumption: one supposes it. But this is not what happens in EUCLID's EPIPHANY. In this case, Euclid unintentionally comes to know some proposition by reductio. He falsely believes $p$, comes to see that $p$ implies $q$ and not- $q$, and inferentially comes to know not- $p$ by appealing to the inference rule of reductio.

Second, the standard response does not seem to help. Euclid falsely believes that there are finitely many primes. But he does not have an additional proxy belief $(i)$ which is in the vicinity of the false belief that there are finitely many primes, (ii) is such that he believes and knows it, and (iii) and is such that from it, he infers that there are infinitely many primes. In the more familiar $\mathrm{KFF} / \mathrm{KDF}$ cases, when $p$ is the relevant false belief, there are true proxies in the vicinity, typically of the form 'Approximately, $p$ '. But this isn't the case in EUCLID's EPIPHANY. Since the false belief that there are finitely many primes is an essential belief in Euclid's inferentially coming to know that there are infinitely many primes, (No FAlse Belief) is false. ${ }^{6}$

I will end by considering two natural objections, both of which fall out of the thought that the case might be under-described. First, just as one who adopts the standard response inserts a proxy into BAD Counting and

\footnotetext{
${ }^{6}$ Two quick notes. First, Schnee (2015) responds to a worry that the standard response might not apply to cases beyond DODGY WATch. He successfully shows, however, that it naturally applies to at least some further cases - for instance, the case from Klein (2008) involving a child believing falsely that Santa will bring gifts and nevertheless coming to know that there will be presents under the tree. The proxy here is 'someone will bring presents'. However, the standard response doesn't help with Euclid's Epiphany. Second, those who endorse KFF sometimes make further claims about the cases - Warfield (2005) says the false belief epistemizes the conclusion, and Klein (2008) says that it doxastically justifies it. These epistemic notions are contentious and I will ignore them here. In this paper, I am merely interested in (No FALSE BELIEF).
} 
DodGy WATCH, one might try to force some proxy into EUCLID's EPIPHANY, perhaps a conditional belief like 'if the proposition that there are finitely many primes implies some proposition $q$ and not- $q$, then the proposition that there are finitely many primes is false'. Admittedly, it is natural to think that in typical cases, when one attempts to prove some proposition not- $p$ by reductio, one also has a conditional belief like 'if $p$ implies a contradiction, then not$p$ '. But this is not what happens to Euclid: Euclid's argument by reductio is unintentional. By forcing a proxy belief into the story, the advocate of the standard response would seem to suggest that one can never unintentionally come to know something by reductio. That seems wrong.

A KFF advocate might try to respond in the following way. For someone to know how to competently argue by reductio, one must at least know the following proposition: for any $p$, if $p$ implies a contradiction, then not- $p$. The KFF advocate might then argue that this proposition provides Euclid with another path to his conclusion. After all, Euclid could have discovered that the proposition that there are finitely many primes implies a contradiction, and then he could have appealed to universal instantiation, together with the conditional above. This might lead one to think that the false belief that there are finitely many primes is not essential.

In response, I should make one point of clarification, and then I will offer what I take to be the main problem with this line of reply. First, the clarification: again, I am taking an essential false belief to be a false belief that figures in all the belief-forming processes that generate the known conclusion. In EUCLID's EPIPHANY, there is one path to Euclid's conclusion: Euclid falsely believed $p$, came to see that $p$ implied a contradiction, and came to know not- $p$, by appealing to reductio. The belief figured in the one and only belief forming process which led to the conclusion and thus is essential. Second, the problem. If the KFF advocate's response is that Euclid cannot inferentially come to know something by reductio without appealing to the proposition 'for all $p$, if $p$ implies a contradiction, then not- $p$ ', then it is hard to see how any inference could rely on essential premises. Consider a simple case of modus ponens. If I know $p$, come to know if $p$ then $q$, and then inferentially come to know $q$ by appealing to modus ponens, must there also be some path to my knowing $q$ which relies on the conditional proposition 'for all $p$, and for all $q$, if $p$ and if $p$ then $q$, then $q$ '? To force this additional conditional belief into my simple inference of modus ponens would render my initial belief 'if $p$ then $q$ ' inessential, and seem to imply that I never make inferences from premises all of which are essential. In this case, it's no longer obvious to me what the KFF advocate means by principles like (No FALSE BELIEF).

It's worth noting that there are other conceptions one might have of an 'essential' false belief. Klein (2008), for instance, proposes a counterfactual test 
for essentiality: "if the false belief were simply removed from the actual causal chain that resulted in knowledge, no causal chain resulting in the cognition would remain" (pg. 41). If we interpret 'essential' counterfactually, as Klein does, Euclid's false belief clearly seems to be essential: if the belief that there are finitely many primes was simply removed from the actual causal chain that resulted in knowledge (that is, the unintended reductio), no causal chain resulting in cognition would seem to remain.

Now for the second objection: even if there is no proxy belief, maybe there is a proxy supposition. That is, one might think that in addition to his false belief that there are finitely many primes, Euclid must also have the mental supposition that there are finitely many primes. After all, in order for Euclid to come to know that there are infinitely many primes, he has to come to know it in the right sort of way. But if there is also an additional supposition somewhere along the way, then the false belief is not essential, since there is another path to the conclusion (namely: the supposition that $p$, the deduction of $q$ and not- $q$, and the inference of not- $p$ ), and thus the false belief is not essential. Certainly, there are cases which one could describe which are distinct but similar to EUCLID's EPIPHANY - for instance, where Euclid falsely believes $p$, derives a contradiction, merely suspends his belief that $p$ (given, say, the shock that this deduction induces on him), supposes that $p$, re-derives the contradiction, and only later comes to know not- $p$ (in fact, maybe he's so shocked at this result, that he must check the proof yet another time before he comes to know the conclusion). Admittedly, amended cases like these are not counterexamples to (No FALSE BELIEF), because the false belief is not essential to Euclid inferentially coming to know his conclusion. My contention, however, is simply that a case like EUCLID's EPIPHANY is possible. It is possible for me to be extremely familiar with reductio, falsely believe $p$, come to see that it implies a contradiction, and thereby come to know not- $p$ in virtue of knowing reductio, without, say, having to recheck my derivation, or additionally suppose my assumption and then re-derive the contradiction. I know merely by reductio. If this is right, then EUCLID's EPIPHANY is a counterexample to (No FALSE BELIEF), and the standard response doesn't seem to help. ${ }^{7}$

\section{REFERENCES}

[1] D.M. Armstrong. Belief, Truth, and Knowledge. Cambridge: Cambridge University Press, 1973.

[2] Brian Ball and Michael Blome-Tillmann. Counter closure and knowledge despite falsehood. Philosophical Quarterly, 64(257):552-68, 2014.

${ }^{7}$ Thanks to Andrew Bacon, John Hawthorne, Frank Hong, Jeffrey Sanford Russell, and Gabriel Uzquiano for helpful feedback. 
[3] Christopher Buford and Christopher Michael Cloos. A dilemma for the knowledge despite falsehood strategy. Episteme, pages 1-17, 2017.

[4] Michael Clark. Knowledge and grounds: A comment on mr. gettier's paper. Analysis, 24(2):46-8, 1963.

[5] Branden Fitelson. Closure, counter-closure, and inferential knowledge. In Claudio de Almeida Rodrigo Borges and Peter D. Klein, editors, Explaining Knowledge: New Essays on the Gettier Problem. Oxford: Oxford University Press, 2018.

[6] John Hawthorne and Dani Rabinowitz. Knowledge from false belief. In Claudio de Almeida Rodrigo Borges and Peter D. Klein, editors, Explaining Knowledge: New Essays on the Gettier Problem. Oxford: Oxford University Press, 2018.

[7] Peter Klein. Useful false beliefs. In Q Smith, editor, Epistemology: New Essays, pages 25-62. Oxford: Oxford University Press, 2008.

[8] Saul Kripke. Philosophical Troubles, volume 1. Oxford: Oxford University Press, 2011.

[9] Federico Luzzi. Counter-closure. Australasian Journal of Philosophy, 88(4):673-83, 2010.

[10] Martin Montminy. Knowledge despite falsehood. Canadian Journal of Philosophy, 44(34):463-475, 2014.

[11] Bertrand Russell. The Problems of Philosophy. Oxford: Oxford University Press, 1912.

[12] Ian Schnee. There is no knowledge from falsehood. Episteme, 12(1):53-74, 2015.

[13] Ted Warfield. Knowledge from falsehood. Philosophical Perspectives, 19:405-16, 2005.

[14] Timothy Williamson. The Philosophy of Philosophy. Oxford: Blackwell, 2007. 